\title{
KAZHDAN'S PROPERTY $T$ FOR DISCRETE QUANTUM GROUPS
}

\author{
PierRe Fima*广
}

\begin{abstract}
We give a simple definition of property $T$ for discrete quantum groups. We prove the basic expected properties: discrete quantum groups with property $T$ are finitely generated and unimodular. Moreover we show that, for "I.C.C." discrete quantum groups, property $T$ is equivalent to Connes' property $T$ for the dual von Neumann algebra. This allows us to give the first example of a property $T$ discrete quantum group which is not a group using the twisting construction.
\end{abstract}

\section{Introduction}

In the 1980's, Woronowicz [19, 20], 21] introduced the notion of a compact quantum group and generalized the classical Peter-Weyl representation theory. Many interesting examples of compact quantum groups are available by now: Drinfel'd and Jimbo [5, 9] introduced q-deformations of compact semi-simple Lie groups, and Rosso [13] showed that they fit into the theory of Woronowicz. Free orthogonal and unitary quantum groups were introduced by Van Daele and Wang [18] and studied in detail by Banica 1], 2].

Some discrete group-like properties and proofs have been generalized to (the dual of) compact quantum groups. See, for example, the work of Tomatsu [14] on amenability, the work of Banica and Vergnioux [3] on growth and the work of Vergnioux and Vaes [15] on boundary.

The aim of this paper is to define property $T$ for discrete quantum groups. We give a definition analogous to the group case using almost invariant vectors. We show that a discrete quantum group with property $\mathrm{T}$ is finitely generated, i.e. the dual is a compact quantum group of matrices. Recall that a locally compact group with property $T$ is unimodular. We show that the same result holds for discrete quantum groups, i.e. every discrete quantum group with property $\mathrm{T}$ is a Kac algebra. In [4 Connes and Jones defined property $T$ for arbitrary von Neumann algebras and showed that an I.C.C. group has property $T$ if and only if its group von Neumann algebra (which is a $\mathrm{II}_{1}$ factor) has property $T$. We show that if the group von Neumann algebra of a discrete quantum group $\widehat{\mathbb{G}}$ is

\footnotetext{
${ }^{*}$ Department of Mathematics, University of Illinois at Urbana-Champaign, Urbana, Illinois 61801, United States. Email: pfima@illinois.edu

${ }^{\dagger}$ This research was supported by the ANR (NCLp).
} 
an infinite dimensional factor (i.e. $\widehat{\mathbb{G}}$ is "I.C.C."), then $\widehat{\mathbb{G}}$ has property $T$ if and only if its group von Neumann algebra is a $\mathrm{II}_{1}$ factor with property $T$. This allows us to construct an example of a discrete quantum group with property $T$ which is not a group by twisting an I.C.C. property $T$ group. In addition we show that free quantum groups do not have property $T$.

This paper is organized as follows: in Section 2 we recall the notions of compact and discrete quantum groups and the main results of this theory. We introduce the notion of discrete quantum sub-groups and prove some basic properties of the quasi-regular representation. We also recall the definition of property $T$ for von Neumann algebras. In Section 3 we introduce property $T$ for discrete quantum groups, we give some basic properties and we show our main result.

\section{Preliminaries}

\section{$2.1 \quad$ Notations}

The scalar product of a Hilbert space $H$, which is denoted by $\langle.,$.$\rangle , is supposed to$ be linear in the first variable. The von Neumann algebra of bounded operators on $H$ will by denoted by $\mathcal{B}(H)$ and the $C^{*}$ algebra of compact operators by $\mathcal{B}_{0}(H)$. We will use the same symbol $\otimes$ to denote the tensor product of Hilbert spaces, the minimal tensor product of $C^{*}$ algebras and the spatial tensor product of von Neumann algebras. We will use freely the leg numbering notation.

\subsection{Compact quantum groups}

We briefly overview the theory of compact quantum groups developed by Woronowicz in 21]. We refer to the survey paper [12] for a smooth approach to these results.

Definition 1. A compact quantum group is a pair $\mathbb{G}=(A, \Delta)$, where $A$ is a unital $C^{*}$ algebra; $\Delta$ is unital *-homomorphism from $A$ to $A \otimes A$ satisfying $(\Delta \otimes$ id $) \Delta=($ id $\otimes \Delta) \Delta$ and $\Delta(A)(A \otimes 1)$ and $\Delta(A)(1 \otimes A)$ are dense in $A \otimes A$.

Notation 1. We denote by $C(\mathbb{G})$ the $C^{*}$ algebra $A$.

The major results in the general theory of compact quantum groups are the existence and uniqueness of the Haar state and the Peter-Weyl representation theory.

Theorem 1. Let $\mathbb{G}$ be a compact quantum group. There exists a unique state $\varphi$ on $C(\mathbb{G})$ such that $(i d \otimes \varphi) \Delta(a)=\varphi(a) 1=(\varphi \otimes i d) \Delta(a)$ for all $a \in C(\mathbb{G})$. The state $\varphi$ is called the Haar state of $\mathbb{G}$.

Notation 2. The Haar state need not be faithful. We denote by $\mathbb{G}_{\text {red }}$ the reduced quantum group obtained by taking $C\left(\mathbb{G}_{\mathrm{red}}\right)=C(\mathbb{G}) / I$ where $I=\{x \in$ $\left.A \mid \varphi\left(x^{*} x\right)=0\right\}$. The Haar measure is faithful on $\mathbb{G}_{\text {red }}$. We denote by $L^{\infty}(\mathbb{G})$ the von Neumann algebra generated by the G.N.S. representation of the Haar state of $\mathbb{G}$. Note that $L^{\infty}\left(\mathbb{G}_{\text {red }}\right)=L^{\infty}(\mathbb{G})$. 
Definition 2. A unitary representation $u$ of a compact quantum group $\mathbb{G}$ on a Hilbert space $H$ is a unitary element $u \in \mathrm{M}\left(\mathcal{B}_{0}(H) \otimes C(\mathbb{G})\right)$ satisfying

$$
(\mathrm{id} \otimes \Delta)(u)=u_{12} u_{13} .
$$

Let $u^{1}$ and $u^{2}$ be two unitary representations of $\mathbb{G}$ on the respective Hilbert spaces $H_{1}$ and $H_{2}$. We define the set of intertwiners

$$
\operatorname{Mor}\left(u^{1}, u^{2}\right)=\left\{T \in \mathcal{B}\left(H_{1}, H_{2}\right) \mid(T \otimes 1) u^{1}=u^{2}(T \otimes 1)\right\} .
$$

A unitary representation $u$ is said to be irreducible if $\operatorname{Mor}(u, u)=\mathbb{C} 1$. Two unitary representations $u^{1}$ and $u^{2}$ are said to be unitarily equivalent if there is a unitary element in $\operatorname{Mor}\left(u^{1}, u^{2}\right)$.

Theorem 2. Every irreducible representation is finite-dimensional. Every unitary representation is unitarily equivalent to a direct sum of irreducibles.

Definition 3. Let $u^{1}$ and $u^{2}$ be unitary representations of $\mathbb{G}$ on the respective Hilbert spaces $H_{1}$ and $H_{2}$. We define the tensor product

$$
u^{1} \otimes u^{2}=u_{13}^{1} u_{23}^{2} \in \mathrm{M}\left(\mathcal{B}_{0}\left(H_{1} \otimes H_{2}\right) \otimes C(\mathbb{G})\right) .
$$

Notation 3. We denote by $\operatorname{Irred}(\mathbb{G})$ the set of (equivalence classes) of irreducible unitary representations of a compact quantum group $\mathbb{G}$. For every $x \in \operatorname{Irred}(\mathbb{G})$ we choose representatives $u^{x}$ on the Hilbert space $H_{x}$. Whenever $x, y \in \operatorname{Irred}(\mathbb{G})$, we use $x \otimes y$ to denote the (class of the) unitary representation $u^{x} \otimes u^{y}$. The class of the trivial representation is denoted by 1 .

The set $\operatorname{Irred}(\mathbb{G})$ is equipped with a natural involution $x \mapsto \bar{x}$ such that $u^{\bar{x}}$ is the unique (up to unitary equivalence) irreducible representation such that

$$
\operatorname{Mor}(1, x \otimes \bar{x}) \neq 0 \neq \operatorname{Mor}(1, \bar{x} \otimes x) .
$$

This means that $x \otimes \bar{x}$ and $\bar{x} \otimes x$ contain a non-zero invariant vector. Let $E_{x} \in H_{x} \otimes H_{\bar{x}}$ be a non-zero invariant vector and $J_{x}$ the invertible antilinear map from $H_{x}$ to $H_{\bar{x}}$ defined by

$$
\left\langle J_{x} \xi, \eta\right\rangle=\left\langle E_{x}, \xi \otimes \eta\right\rangle, \quad \text { for all } \xi \in H_{x}, \eta \in H_{\bar{x}} .
$$

Let $Q_{x}=J_{x}^{*} J_{x}$. We will always choose $E_{x}$ and $E_{\bar{x}}$ normalized such that $\left\|E_{x}\right\|=$ $\left\|E_{\bar{x}}\right\|$ and $J_{\bar{x}}=J_{x}^{-1}$. Then $Q_{x}$ is uniquely determined, $\operatorname{Tr}\left(Q_{x}\right)=\left\|E_{x}\right\|^{2}=$ $\operatorname{Tr}\left(Q_{x}^{-1}\right)$ and $Q_{\bar{x}}=\left(J_{x} J_{x}^{*}\right)^{-1}$. $\operatorname{Tr}\left(Q_{x}\right)$ is called the quantum dimension of $x$ and is denoted by $\operatorname{dim}_{\mathrm{q}}(x)$. The unitary representation $u^{\bar{x}}$ is called the contragredient of $u^{x}$.

The G.N.S. representation of the Haar state is given by $\left(L^{2}(\mathbb{G}), \Omega\right)$ where $L^{2}(\mathbb{G})=\bigoplus_{x \in \operatorname{Irred}(\mathbb{G})} H_{x} \otimes H_{\bar{x}}, \Omega \in H_{1} \otimes H_{\overline{1}}$ is the unique norm one vector, and

$$
\left(\omega_{\xi, \eta} \otimes \mathrm{id}\right)\left(u^{x}\right) \Omega=\frac{1}{\left\|E_{x}\right\|} \xi \otimes J_{x}(\eta), \quad \text { for all } \xi, \eta \in H_{x} .
$$

It is easy to see that $\varphi$ is a trace if and only if $Q_{x}=$ id for all $x \in \operatorname{Irred}(\mathbb{G})$. In this case $\left\|E_{x}\right\|=\sqrt{n_{x}}$ where $n_{x}$ is the dimension of $H_{x}$ and $J_{x}$ is an anti-unitary operator. 
Notation 4. Let $C(\mathbb{G})_{s}$ be the vector space spanned by the coefficients of all irreducible representations of $\mathbb{G}$. Then $C(\mathbb{G})_{s}$ is a dense unital *-subalgebra of $C(\mathbb{G})$. Let $C\left(\mathbb{G}_{\max }\right)$ be the maximal $C^{*}$ completion of the unital *-algebra $C(\mathbb{G})_{s} . C\left(\mathbb{G}_{\max }\right)$ has a canonical structure of a compact quantum group. This quantum group is denoted by $\mathbb{G}_{\max }$ and it is called the maximal quantum group.

A morphism of compact quantum groups $\pi: \mathbb{G} \rightarrow \mathbb{H}$ is a unital *-homomorphism from $C\left(\mathbb{G}_{\max }\right)$ to $C\left(\mathbb{H}_{\max }\right)$ such that $\Delta_{\mathbb{H}} \circ \pi=(\pi \otimes \pi) \circ \Delta_{\mathbb{G}}$, where $\Delta_{\mathbb{G}}$ and $\Delta_{\mathbb{H}}$ denote the comultiplications for $\mathbb{G}_{\max }$ and $\mathbb{H}_{\max }$ respectively. We will need the following easy Lemma.

Lemma 1. Let $\pi$ be a surjective morphism of compact quantum group from $\mathbb{G}$ to $\mathbb{H}$ and $\tilde{\pi}$ be the surjective *-homomorphism from $C\left(\mathbb{G}_{\max }\right)$ to $C(\mathbb{H})$ obtained by composition of $\pi$ with the canonical surjection $C\left(\mathbb{H}_{\text {max }}\right) \rightarrow C(\mathbb{H})$. Then for every irreducible unitary representation $v$ of $\mathbb{H}$ there exists an irreducible unitary representation $u$ of $\mathbb{G}$ such that $v$ is contained in the unitary representation $(i d \otimes \tilde{\pi})(u)$.

Proof. Let $\varphi$ be the Haar state of $\mathbb{H}$ and $v$ be an irreducible unitary representation of $\mathbb{H}$ on the Hilbert space $H_{v}$. Because $v$ is irreducible it is sufficient to show that there exists a unitary irreducible representation $u$ of $\mathbb{G}$ such that $\operatorname{Mor}(w, v) \neq\{0\}$, where $w=(\operatorname{id} \otimes \tilde{\pi})(u)$. Suppose that the statement is false. Then for all irreducible unitary representations $u$ of $\mathbb{G}$ on $H_{u}$, we have $\operatorname{Mor}(w, v)=\{0\}$. By [12, Lemma 6.3, for every operator $a: H_{v} \rightarrow H_{u}$ the operator $($ id $\otimes \varphi)\left(v^{*}(a \otimes 1) w\right)$ is in $\operatorname{Mor}(w, v)$. It follows that for every irreducible unitary representation $u$ of $\mathbb{G}$ and every operator $a: H_{v} \rightarrow H_{u}$ we have (id $\otimes \varphi)\left(v^{*}(a \otimes 1) w\right)=0$. Using the same techniques as in [12], Theorem 6.7 , (because, by the surjectivity of $\pi, \tilde{\pi}\left(C(\mathbb{G})_{s}\right)$ is dense in $C(\mathbb{H})$ ) we find $($ id $\otimes \varphi)\left(v^{*} v\right)=0$. But this is a contradiction as $v^{*} v=1$.

The collection of all finite-dimensional unitary representations (given with the concrete Hilbert spaces) of a compact quantum group $\mathbb{G}$ is a complete concrete monoidal $W^{*}$-category. We denote this category by $\mathcal{R}(\mathbb{G})$. We say that $\mathcal{R}(\mathbb{G})$ is finitely generated if there exists a finite subset $E \subset \operatorname{Irred}(\mathbb{G})$ such that for all finite-dimensional unitary representations $r$ there exists a finite family of morphisms $b_{k} \in \operatorname{Mor}\left(r_{k}, r\right)$, where $r_{k}$ is a product of elements of $E$, and $\sum_{k} b_{k} b_{k}^{*}=I_{r}$. It is not difficult to show that $\mathcal{R}(\mathbb{G})$ is finitely generated if and only if $\mathbb{G}$ is a compact quantum group of matrices (see [20]).

\subsection{Discrete quantum groups}

A discrete quantum group is defined as the dual of a compact quantum group.

Definition 4. Let $\mathbb{G}$ be a compact quantum group. We define the dual discrete quantum group $\widehat{\mathbb{G}}$ as follows:

$$
c_{0}(\widehat{\mathbb{G}})=\bigoplus_{x \in \operatorname{Irred}(\mathbb{G})}^{c_{0}} \mathcal{B}\left(H_{x}\right), \quad l^{\infty}(\widehat{\mathbb{G}})=\bigoplus_{x \in \operatorname{Irred}(\mathbb{G})}^{\infty} \mathcal{B}\left(H_{x}\right) .
$$


We denote the minimal central projection of $l^{\infty}(\widehat{\mathbb{G}})$ by $p_{x}, x \in \operatorname{Irred}(\mathbb{G})$. We have a natural unitary $\mathbb{V} \in \mathrm{M}\left(c_{o}(\widehat{\mathbb{G}}) \otimes C(\mathbb{G})\right)$ given by

$$
\mathbb{V}=\bigoplus_{x \in \operatorname{Irred}(\mathbb{G})} u^{x}
$$

We have a natural comultiplication

$$
\hat{\Delta}: l^{\infty}(\widehat{\mathbb{G}}) \rightarrow l^{\infty}(\widehat{\mathbb{G}}) \otimes l^{\infty}(\widehat{\mathbb{G}}):(\hat{\Delta} \otimes i d)(\mathbb{V})=\mathbb{V}_{13} \mathbb{V}_{23} .
$$

The comultiplication is given by the following formula

$$
\hat{\Delta}(a) S=S a, \quad \text { for all } a \in \mathcal{B}\left(H_{x}\right), S \in \operatorname{Mor}(x, y z), x, y, z \in \operatorname{Irred}(\mathbb{G}) .
$$

Remark 1. The maximal and reduced versions of a compact quantum group are different versions of the same underlying compact quantum group. This different versions give the same dual discrete quantum group, i.e. $\widehat{\mathbb{G}}=\widehat{\mathbb{G}_{\text {red }}}=\widehat{\mathbb{G}_{\max }}$. This means that $\widehat{\mathbb{G}}, \widehat{\mathbb{G}_{\text {red }}}$ and $\widehat{\mathbb{G}_{\max }}$ have the same $C^{*}$ algebra, the same von Neumann algebra and the same comultiplication.

A morphism of discrete quantum groups $\hat{\pi}: \widehat{\mathbb{G}} \rightarrow \widehat{\mathbb{H}}$ is a non-degenerate *-homomorphism from $c_{0}(\widehat{\mathbb{G}})$ to $\mathrm{M}\left(c_{0}(\widehat{\mathbb{H}})\right)$ such that $\hat{\Delta}_{\mathbb{H}} \circ \pi=(\pi \otimes \pi) \circ \hat{\Delta}_{\mathbb{G}}$, where $\hat{\Delta}_{\mathbb{G}}$ and $\hat{\Delta}_{\mathbb{H}}$ denote the comultiplication for $\widehat{\mathbb{G}}$ and $\widehat{\mathbb{H}}$ respectively. Every morphism of compact quantum groups $\pi: \mathbb{G} \rightarrow \mathbb{H}$ admits a canonical dual morphism of discrete quantum groups $\hat{\pi}: \widehat{\mathbb{G}} \rightarrow \widehat{\mathbb{H}}$. Conversely, every morphism of discrete quantum groups $\hat{\pi}: \widehat{\mathbb{G}} \rightarrow \widehat{\mathbb{H}}$ admits a canonical dual morphism of compact quantum groups $\pi: \mathbb{G} \rightarrow \mathbb{H}$. Moreover, $\pi$ is surjective (resp. injective) if and only if $\hat{\pi}$ is injective (resp. surjective).

We say that a discrete quantum group $\widehat{\mathbb{G}}$ is finitely generated if the category $\mathcal{R}(\mathbb{G})$ is finitely generated.

We will work with representations in the von Neumann algebra setting.

Definition 5. Let $\widehat{\mathbb{G}}$ be a discrete quantum group. A unitary representation $U$ of $\widehat{\mathbb{G}}$ on a Hilbert space $H$ is a unitary $U \in l^{\infty}(\widehat{\mathbb{G}}) \otimes \mathcal{B}(H)$ such that :

$$
(\hat{\Delta} \otimes \mathrm{id})(U)=U_{13} U_{23} .
$$

Consider the following maximal version of the unitary $\mathbb{V}$ :

$$
\mathcal{V}=\bigoplus_{x \in \operatorname{Irred}(\mathbb{G})} u^{x} \in \mathrm{M}\left(c_{o}(\widehat{\mathbb{G}}) \otimes C\left(\mathbb{G}_{\max }\right)\right)
$$

For every unitary representation $U$ of $\widehat{\mathbb{G}}$ on a Hilbert space $H$ there exists a unique ${ }^{*}$-homomorphism $\rho: C\left(\mathbb{G}_{\max }\right) \rightarrow \mathcal{B}(H)$ such that $($ id $\otimes \rho)(\mathcal{V})=U$.

Notation 5. Whenever $U$ is a unitary representation of $\widehat{\mathbb{G}}$ on a Hilbert space $H$ we write $U=\sum_{x \in \operatorname{Irred}(\mathbb{G})} U^{x}$ where $U^{x}=U p_{x}$ is a unitary in $\mathcal{B}\left(H_{x}\right) \otimes \mathcal{B}(H)$. 
The discrete quantum group $l^{\infty}(\widehat{\mathbb{G}})$ comes equipped with a natural modular structure. Let us define the following canonical states on $\mathcal{B}\left(H_{x}\right)$ :

$$
\varphi_{x}(A)=\frac{\operatorname{Tr}\left(Q_{x} A\right)}{\operatorname{Tr}\left(Q_{x}\right)}, \quad \text { and } \quad \psi_{x}(A)=\frac{\operatorname{Tr}\left(Q_{x}^{-1} A\right)}{\operatorname{Tr}\left(Q_{x}^{-1}\right)}, \quad \text { for all } A \in \mathcal{B}\left(H_{x}\right) .
$$

The states $\varphi_{x}$ and $\psi_{x}$ provide a formula for the invariant normal semi-finite faithful (n.s.f.) weights on $l^{\infty}(\widehat{\mathbb{G}})$.

Proposition 1. The left invariant weight $\hat{\varphi}$ and the right invariant weight $\hat{\psi}$ on $\widehat{\mathbb{G}}$ are given by

$$
\hat{\varphi}(a)=\sum_{x \in \operatorname{Irred}(\mathbb{G})} \operatorname{dim}_{q}(x)^{2} \varphi_{x}\left(a p_{x}\right) \quad \text { and } \quad \hat{\psi}(a)=\sum_{x \in \operatorname{Irred}(\mathbb{G})} \operatorname{dim}_{q}(x)^{2} \psi_{x}\left(a p_{x}\right),
$$

for all $a \in l^{\infty}(\widehat{\mathbb{G}})$ whenever this formula makes sense.

A discrete quantum is unimodular (i.e. the left and right invariant weights are equal) if and only if the Haar state $\varphi$ on the dual is a trace. In general, a discrete quantum group is not unimodular, and it is easy to check that the Radon-Nikodym derivative is given by

$$
[D \hat{\psi}: D \hat{\varphi}]_{t}=\hat{\delta}^{i t} \quad \text { where } \quad \hat{\delta}=\sum_{x \in \operatorname{Irred}(\mathbb{G})} Q_{x}^{-2} p_{x} .
$$

The positive self-adjoint operator $\hat{\delta}$ is called the modular element: it is affiliated with $c_{0}(\widehat{\mathbb{G}})$ and satisfies $\hat{\Delta}(\hat{\delta})=\hat{\delta} \otimes \hat{\delta}$.

The following Proposition is very easy to prove.

Proposition 2. Let $\Gamma$ be the subset of $\mathbb{R}_{+}^{*}$ consisting of all the eigenvalues of the operators $Q_{x}^{-2}$ for $x \in \operatorname{Irred}(\mathbb{G})$. Then $\Gamma$ is a subgroup of $\mathbb{R}_{+}^{*}$ and $\operatorname{Sp}(\widehat{\delta})=\Gamma \cup\{0\}$.

Proof. Note that, because $J_{\bar{x}}=J_{x}^{-1}$, the eigenvalues of $Q_{\bar{x}}$ are the inverse of the eigenvalues of $Q_{x}$. Using the formula $S Q_{z}=Q_{x} \otimes Q_{y} S$, when $z \subset x \otimes y$ and $S \in \operatorname{Mor}(z, x \otimes y)$ is an isometry, the Proposition follows immediately.

\subsection{Discrete quantum subgroups}

Let $\mathbb{G}$ be a compact quantum group with representation category $\mathcal{C}$. Let $\mathcal{D}$ be a full subcategory such that $1_{\mathcal{C}} \in \mathcal{D}, \mathcal{D} \otimes \mathcal{D} \subset \mathcal{D}$ and $\overline{\mathcal{D}}=\mathcal{D}$. By the TannakaKrein Reconstruction Theorem of Woronowicz [20] we know that there exists a compact quantum group $\mathbb{H}$ such that the representation category of $\mathbb{H}$ is $\mathcal{D}$. We say that $\mathbb{\mathbb { H }}$ is a discrete quantum subgroup of $\widehat{\mathbb{G}}$. We have $\operatorname{Irred}(\mathbb{H}) \subset \operatorname{Irred}(\mathbb{G})$. We collect some easy observations in the next proposition. We denote by a subscript $\mathbb{H}$ the objects associated to $\mathbb{H}$. 
Proposition 3. Let $p=\sum_{x \in \operatorname{Irred}(\mathbb{H})} p_{x}$. We have:

1. $\hat{\Delta}(p)(p \otimes 1)=p \otimes p ;$

2. $l^{\infty}(\widehat{\mathbb{H}})=p\left(l^{\infty}(\widehat{\mathbb{G}})\right)$;

3. $\hat{\Delta}_{\mathbb{H}}(a)=\hat{\Delta}(a)(p \otimes p)$ for all $a \in l^{\infty}(\widehat{\mathbb{H}})$;

4. $\hat{\varphi}(p$. $)=\hat{\varphi}_{\mathbb{H}}$ and $\hat{\delta}_{\mathbb{H}}=p \hat{\delta}$.

Proof. For $x, y, z \in \operatorname{Irred}(\mathbb{G})$ such that $y \subset z \otimes x$, we denote by $p_{y}^{z \otimes x} \in \operatorname{End}(x \otimes y)$ the projection on the sum of all sub-representations equivalent to $y$. Note that

$$
\hat{\Delta}\left(p_{y}\right)\left(p_{z} \otimes p_{x}\right)= \begin{cases}p_{y}^{z \otimes x} & \text { if } y \subset z \otimes x \\ 0 & \text { otherwise }\end{cases}
$$

Thus:

$$
\hat{\Delta}(p)\left(p_{z} \otimes p_{x}\right)=\sum_{y \in \operatorname{Irred}(\mathbb{H}), y \subset z \otimes x} p_{y}^{z \otimes x} .
$$

Note that if $y \subset z \otimes x$ and $y, z \in \operatorname{Irred}(\mathbb{H})$ then $x \in \operatorname{Irred}(\mathbb{H})$. It follows that:

$$
\hat{\Delta}(p)\left(p \otimes p_{x}\right)= \begin{cases}p \otimes p_{x} & \text { if } x \in \operatorname{Irred}(\mathbb{H}), \\ 0 & \text { otherwise }\end{cases}
$$

Thus, $\hat{\Delta}(p)(p \otimes 1)=p \otimes p$. The other assertions are obvious.

We introduce the following equivalence relation on $\operatorname{Irred}(\mathbb{G})$ (see [17): if $x, y \in \operatorname{Irred}(\mathbb{G})$ then $x \sim y$ if and only if there exists $t \in \operatorname{Irred}(\mathbb{H})$ such that $x \subset y \otimes t$. We define the right action of $\widehat{\mathbb{H}}$ on $l^{\infty}(\widehat{\mathbb{G}})$ by translation:

$$
\alpha: l^{\infty}(\widehat{\mathbb{G}}) \rightarrow l^{\infty}(\widehat{\mathbb{G}}) \otimes l^{\infty}(\widehat{\mathbb{H}}), \quad \alpha(a)=\hat{\Delta}(a)(1 \otimes p) .
$$

Using $\hat{\Delta}(p)(p \otimes 1)=p \otimes p$ and $\hat{\Delta}_{\mathbb{H}}=\hat{\Delta}().(p \otimes p)$ it is easy to see that $\alpha$ satisfies the following equations:

$$
(\alpha \otimes \mathrm{id}) \alpha=\left(\mathrm{id} \otimes \hat{\Delta}_{\mathbb{H}}\right) \alpha \quad \text { and } \quad(\hat{\Delta} \otimes \mathrm{id}) \alpha=(\mathrm{id} \otimes \alpha) \hat{\Delta} .
$$

The first equality means that $\alpha$ is a right action of $\widehat{\mathbb{H}}$ on $l^{\infty}(\widehat{\mathbb{G}})$. Let $l^{\infty}(\widehat{\mathbb{G}} / \widehat{\mathbb{H}})$ be the set of fixed points of the action $\alpha$ :

$$
l^{\infty}(\widehat{\mathbb{G}} / \widehat{\mathbb{H}}):=\left\{a \in l^{\infty}(\widehat{\mathbb{G}}), \mid \alpha(a)=a \otimes 1\right\} .
$$

Using the second equality for $\alpha$ it is easy to see that:

$$
\hat{\Delta}\left(l^{\infty}(\widehat{\mathbb{G}} / \widehat{\mathbb{H}})\right) \subset l^{\infty}(\widehat{\mathbb{G}}) \otimes l^{\infty}(\widehat{\mathbb{G}} / \widehat{\mathbb{H}}) .
$$

Thus the restriction of $\hat{\Delta}$ to $l^{\infty}(\widehat{\mathbb{G}} / \widehat{\mathbb{H}})$ gives an action of $\widehat{\mathbb{G}}$ on $l^{\infty}(\widehat{\mathbb{G}} / \widehat{\mathbb{H}})$. We denote this action by $\beta$. 
Proposition 4. Let $T_{\alpha}=\left(i d \otimes \hat{\varphi}_{\mathbb{H}}\right) \alpha$ be the normal faithful operator valued weight from $l^{\infty}(\widehat{\mathbb{G}})$ to $l^{\infty}(\widehat{\mathbb{G}} / \widehat{\mathbb{H}})$ associated to $\alpha . T_{\alpha}$ is semi-finite and there exists a unique n.s.f. weight $\theta$ on $l^{\infty}(\widehat{\mathbb{G}} / \widehat{\mathbb{H}})$ such that $\hat{\varphi}=\theta \circ T_{\alpha}$.

Proof. It follows from Eq. (1) that $T_{\alpha}\left(p_{y}\right) p_{z}=0$ if $z \nsim y$. Take $z \sim y$, we have:

$$
\begin{aligned}
T_{\alpha}\left(p_{y}\right) p_{z} & =\sum_{x \in \operatorname{Irred}(\mathbb{H})} \operatorname{dim}_{q}(x)^{2}\left(\mathrm{id} \otimes \varphi_{x}\right)\left(p_{y}^{z \otimes x}\right) \\
& \leq \sum_{x \in \operatorname{Irred}(\mathbb{G})} \operatorname{dim}_{q}(x)^{2}\left(\mathrm{id} \otimes \varphi_{x}\right)\left(p_{y}^{z \otimes x}\right) \\
& =(\operatorname{id} \otimes \hat{\varphi})\left(\hat{\Delta}\left(p_{y}\right)\right) p_{z}=\hat{\varphi}\left(p_{y}\right) p_{z} \\
& =\operatorname{dim}_{q}(y)^{2} p_{z} .
\end{aligned}
$$

It follows that $T_{\alpha}\left(p_{y}\right)<\infty$ for all $y$. This implies that $T_{\alpha}$ is semi-finite. Note that $\alpha\left(\delta^{-i t}\right)=\delta^{-i t} \otimes \delta_{\mathbb{H}}^{-i t}$. It follows from [10, Proposition 8.7, that there exists a unique n.s.f. weight $\theta$ on $l^{\infty}(\widehat{\mathbb{G}} / \widehat{\mathbb{H}})$ such that $\hat{\varphi}=\theta \circ T_{\alpha}$.

Denote by $l^{2}(\widehat{\mathbb{G}} / \widehat{\mathbb{H}})$ the G.N.S. space of $\theta$ and suppose that $l^{\infty}(\widehat{\mathbb{G}} / \widehat{\mathbb{H}}) \subset$ $\mathcal{B}\left(l^{2}(\widehat{\mathbb{G}} / \widehat{\mathbb{H}})\right)$. Let $U^{*} \in l^{\infty}(\widehat{\mathbb{G}}) \otimes \mathcal{B}\left(l^{2}(\widehat{\mathbb{G}} / \widehat{\mathbb{H}})\right)$ be the unitary implementation of $\beta$ associated to $\theta$ in the sense of [16]. Then $U$ is a unitary representation of $\widehat{\mathbb{G}}$ on $l^{2}(\widehat{\mathbb{G}} / \widehat{\mathbb{H}})$ and $\beta(x)=U^{*}(1 \otimes x) U$. We call $U$ the quasi-regular representation of $\widehat{\mathbb{G}}$ modulo $\widehat{\mathbb{H}}$.

Lemma 2. We have $p \in l^{\infty}(\widehat{\mathbb{G}} / \widehat{\mathbb{H}}) \cap \mathcal{N}_{\theta}$. Put $\xi=\Lambda_{\theta}(p)$. If $\widehat{\mathbb{G}}$ is unimodular then $U^{x} \eta \otimes \xi=\eta \otimes \xi$ for all $x \in \operatorname{Irred}(\mathbb{H})$ and all $\eta \in H_{x}$.

Proof. Using $\hat{\Delta}\left(p_{1}\right)\left(1 \otimes p_{x}\right)=p_{1}^{\bar{x} \otimes x}$ it is easy to see that $T_{\alpha}\left(p_{1}\right)=p$. It follows that $p \in l^{\infty}(\widehat{\mathbb{G}} / \widehat{\mathbb{H}})$ and $\theta(p)=\hat{\varphi}\left(p_{1}\right)=1$. Thus $p \in \mathcal{N}_{\theta}$. Let $x \in M^{+}$such that $T_{\alpha}(x)<\infty, \omega \in l^{\infty}(\widehat{\mathbb{G}})_{*}^{+}$and $\mu$ a n.s.f. weight on $l^{\infty}(\widehat{\mathbb{G}} / \widehat{\mathbb{H}})$. Using $(\hat{\Delta} \otimes$ id $) \alpha=($ id $\otimes \alpha) \hat{\Delta}$ we find:

$$
\begin{aligned}
(\omega \otimes \mu) \beta\left(T_{\alpha}(x)\right) & =(\omega \otimes \mu) \hat{\Delta}\left(T_{\alpha}(x)\right)=(\omega \otimes \mu) \hat{\Delta}\left(\left(\mathrm{id} \otimes \hat{\varphi}_{\mathbb{H}}\right)(\alpha(x))\right) \\
& =\left(\omega \otimes \mu \otimes \hat{\varphi}_{\mathbb{H}}\right)((\hat{\Delta} \otimes \mathrm{id}) \alpha(x)) \\
& =\left(\omega \otimes \mu \otimes \hat{\varphi}_{\mathbb{H}}\right)((\mathrm{id} \otimes \alpha) \hat{\Delta}(x)) \\
& =\left(\omega \otimes \mu \circ T_{\alpha}\right) \hat{\Delta}(x) .
\end{aligned}
$$

It follows that, for all $\omega \in l^{\infty}(\widehat{\mathbb{G}})_{*}^{+}$and all $y \in l^{\infty}(\widehat{\mathbb{G}})^{+}$such that $T_{\alpha}(y)<\infty$, we have:

$$
(\omega \otimes \theta) \beta\left(T_{\alpha}(y)\right)=(\omega \otimes \hat{\varphi})(\hat{\Delta}(y))=\hat{\varphi}(y) \omega(1)=\theta\left(T_{\alpha}(y)\right) \omega(1) .
$$

Let $x \in l^{\infty}(\widehat{\mathbb{G}} / \widehat{\mathbb{H}})^{+}$. Because $T_{\alpha}$ is a faithful and semi-finite, there exists an increasing net of positive elements $y_{i}$ in $l^{\infty}(\widehat{\mathbb{G}})^{+}$such that $T_{\alpha}\left(y_{i}\right)<\infty$ for all $i$ and $\operatorname{Sup}_{i}\left(T_{\alpha}\left(y_{i}\right)\right)=x$. It follows that:

$$
(\omega \otimes \theta) \beta(x)=\operatorname{Sup}\left((\omega \otimes \theta) \beta\left(T_{\alpha}\left(y_{i}\right)\right)\right)=\operatorname{Sup}\left(\theta\left(T_{\alpha}\left(y_{i}\right)\right)\right) \omega(1)=\theta(x) \omega(1),
$$


for all $\omega \in l^{\infty}(\widehat{\mathbb{G}})_{*}^{+}$. This means that $\theta$ is $\beta$-invariant. Using this invariance we define the following isometry:

$$
V^{*}\left(\hat{\Lambda}(x) \otimes \Lambda_{\theta}(y)\right)=\left(\hat{\Lambda} \otimes \Lambda_{\theta}\right)(\beta(y)(x \otimes 1)) .
$$

Because $\widehat{\mathbb{G}}$ is unimodular we know from [16, Proposition 4.3 , that $V^{*}$ is the unitary implementation of $\beta$ associated to $\theta$ i.e. $V=U$. Using $\hat{\Delta}(p)(p \otimes 1)=$ $p \otimes p$, it follows that, for all $x \in \mathcal{N}_{\hat{\varphi}}$, we have:

$$
U^{*}\left(p \hat{\Lambda}(x) \otimes \Lambda_{\theta}(p)\right)=\left(\hat{\Lambda} \otimes \Lambda_{\theta}\right)(\hat{\Delta}(p)(p x \otimes 1))=p \hat{\Lambda}(x) \otimes \Lambda_{\theta}(p) .
$$

This concludes the proof.

Remark 2. For general discrete quantum groups it can be proved, as in [6], Théorème 2.9, that $V^{*}$ is a unitary implementing the action $\beta$ and, as in [16, Proposition 4.3 , that $V^{*}$ is the unitary implementation of $\beta$ associated to $\theta$. Thus the previous lemma is also true for general discrete quantum groups.

Lemma 3. Suppose that $U$ has a non-zero invariant vector $\xi \in l^{2}(\widehat{\mathbb{G}} / \widehat{\mathbb{H}})$. Then $\operatorname{Irred}(\mathbb{G}) / \operatorname{Irred}(\mathbb{H})$ is a finite set.

Proof. Let $\xi \in l^{2}(\widehat{\mathbb{G}} / \widehat{\mathbb{H}})$ be a normalized $U$-invariant vector. Using $\beta(x)=$ $U^{*}(1 \otimes x) U$ it is easy to see that $\omega_{\xi}$ is a $\beta$-invariant normal state on $l^{\infty}(\widehat{\mathbb{G}} / \widehat{\mathbb{H}})$, i.e. $\left(\right.$ id $\left.\otimes \omega_{\xi}\right) \beta(x)=\omega_{\xi}(x) 1$ for all $x \in l^{\infty}(\widehat{\mathbb{G}} / \widehat{\mathbb{H}})$. Let $s$ be the support of $\omega_{\xi}$ and $e=1-s$. Let $\omega$ be a faithful normal state on $l^{\infty}(\widehat{\mathbb{G}})$. Because the support of $\omega \otimes \omega_{\xi}$ is $1 \otimes s$ and $\left(\omega \otimes \omega_{\xi}\right) \beta(e)=\omega_{\xi}(e)=0$ we find $\hat{\Delta}(e)=\beta(e) \leq 1 \otimes e$. It follows from [11, Lemma 6.4, that $e=0$ or $e=1$. Because $\xi$ is a non-zero vector we have $e=0$. Thus $\omega_{\xi}$ is faithful. Let $x \in M^{+}$such that $T_{\alpha}(x)<\infty$. By Eq. (2) we have:

$$
\left(\omega \otimes \omega_{\xi} \circ T_{\alpha}\right)(\hat{\Delta}(x))=\left(\omega \otimes \omega_{\xi}\right) \beta\left(T_{\alpha}(x)\right)=\omega_{\xi}\left(T_{\alpha}(x)\right) \omega(1),
$$

for all $\omega \in l^{\infty}(\widehat{\mathbb{G}})_{*}^{+}$. Because $T_{\alpha}$ is n.s.f., it follows easily that $\omega_{\xi} \circ T_{\alpha}$ is a left invariant n.s.f. weight on $\widehat{\mathbb{G}}$. Thus, up to a positive constant, we have $\omega_{\xi} \circ T_{\alpha}=\hat{\varphi}$.

Suppose that $\operatorname{Irred}(\mathbb{G}) / \operatorname{Irred}(\mathbb{H})$ is infinite, and let $x_{i} \in \operatorname{Irred}(\mathbb{G}), i \in \mathbb{N}$ be a complete set of representatives of $\operatorname{Irred}(\mathbb{G}) / \operatorname{Irred}(\mathbb{H})$. Let $a$ be the positive element of $l^{\infty}(\widehat{\mathbb{G}})$ defined by $a=\sum_{i \geq 0} \frac{1}{\operatorname{dim}_{q}\left(x_{i}\right)^{2}} p_{x_{i}}$. Then we have $\hat{\varphi}(a)=+\infty$ and $T_{\alpha}(a)=\sum_{i} \sum_{x \simeq x_{i}} p_{x}=1<\infty$, which is a contradiction.

\subsection{Property $T$ for von Neumann algebras}

Here we recall several facts from [4]. If $M$ and $N$ are von Neumann algebras then a correspondence from $M$ to $N$ is a Hilbert space $H$ which is both a left $M$-module and a right $N$-module, with commuting normal actions $\pi_{l}$ and $\pi_{r}$ respectively. The triple $\left(H, \pi_{l}, \pi_{r}\right)$ is simply denoted by $H$ and we shall write $a \xi b$ instead of $\pi_{l}(a) \pi_{r}(b) \xi$ for $a \in M, b \in N$ and $\xi \in H$. We shall denote by 
$\mathcal{C}(M)$ the set of unitary equivalence classes of correspondences from $M$ to $M$. The standard representation of $M$ defines an element $L^{2}(M)$ of $\mathcal{C}(M)$, called the identity correspondence.

Given $H \in \mathcal{C}(M), \epsilon>0, \xi_{1}, \ldots, \xi_{n} \in H, a_{1}, \ldots, a_{p} \in M$, let $\mathcal{V}_{H}\left(\epsilon, \xi_{i}, a_{i}\right)$ be the set of $K \in \mathcal{C}(M)$ for which there exist $\eta_{1}, \ldots, \eta_{n} \in K$ with

$$
\left|\left\langle a_{j} \eta_{i} a_{k}, \eta_{i^{\prime}}\right\rangle-\left\langle a_{j} \xi_{i} a_{k}, \xi_{i^{\prime}}\right\rangle\right|<\epsilon, \text { for all } i, i^{\prime}, j, k .
$$

Such sets form a basis of a topology on $\mathcal{C}(M)$ and, following [4, $M$ is said to have property $T$ if there is a neighbourhood of the identity correspondence, each member of which contains $L^{2}(M)$ as a direct summand.

When $M$ is a $\mathrm{II}_{1}$ factor the property $T$ is easier to understand. A $\mathrm{II}_{1}$ factor $M$ has property $T$ if we can find $\epsilon>0$ and $a_{1}, \ldots, a_{p} \in M$ satisfying the following condition: every $H \in \mathcal{C}(M)$ such that there exists $\xi \in H,\|\xi\|=1$, with $\left\|a_{i} \xi-\xi a_{i}\right\|<\epsilon$ for all $i$, contains a non-zero central vector $\eta$ (i.e. $a \eta=\eta a$ for all $a \in M)$. We recall the following Proposition from [4.

Proposition 5. If $M$ is a $\mathrm{II}_{1}$ factor with property $T$ then there exist $\epsilon>0$, $b_{1}, \ldots, b_{m} \in M$ and $C>0$ with the following property: for any $\delta \leq \epsilon$, if $H \in \mathcal{C}(M)$ and $\xi \in H$ is a unit vector satisfying $\left\|b_{i} \xi-\xi b_{i}\right\|<\delta$ for all $1 \leq i \leq m$, then there exists a unit central vector $\eta \in H$ such that $\|\xi-\eta\|<C \delta$.

It is proved in 4 that a discrete I.C.C. group has property $T$ if and only if the group von Neumann algebra $\mathcal{L}(G)$ has property $T$.

\section{Property $T$ for Discrete Quantum Groups}

Definition 6. Let $\widehat{\mathbb{G}}$ be a discrete quantum group.

- Let $E \subset \operatorname{Irred}(\mathbb{G})$ be a finite subset, $\epsilon>0$ and $U$ a unitary representation of $\widehat{\mathbb{G}}$ on a Hilbert space $K$. We say that $U$ has an $(E, \epsilon)$-invariant vector if there exists a unit vector $\xi \in K$ such that for all $x \in E$ and $\eta \in H_{x}$ we have:

$$
\left\|U^{x} \eta \otimes \xi-\eta \otimes \xi\right\|<\epsilon\|\eta\| .
$$

- We say that $U$ has almost invariant vectors if, for all finite subsets $E \subset$ $\operatorname{Irred}(\mathbb{G})$ and all $\epsilon>0, U$ has an $(E, \epsilon)$-invariant vector.

- We say that $\widehat{\mathbb{G}}$ has property $T$ if every unitary representation of $\hat{\mathbb{G}}$ having almost invariant vectors has a non-zero invariant vector.

Remark 3. Let $\mathbb{G}=\left(C^{*}(\Gamma), \Delta\right)$, where $\Gamma$ is a discrete group and $\Delta(g)=g \otimes g$ for $g \in \Gamma$. It follows from the definition that $\widehat{\mathbb{G}}$ has property $T$ if and only if $\Gamma$ has property $T$.

The next proposition will be useful to show that the dual of a free quantum group does not have property $T$. 
Proposition 6. Let $\mathbb{G}$ and $\mathbb{H}$ be compact quantum groups. Suppose that there is a surjective morphism of compact quantum groups from $\mathbb{G}$ to $\mathbb{H}$ (or an injective morphism of discrete quantum groups from $\widehat{\mathbb{H}}$ to $\widehat{\mathbb{G}})$. If $\widehat{\mathbb{G}}$ has property $T$ then $\widehat{\mathbb{H}}$ has property $T$.

Proof. We can suppose that $\mathbb{G}=\mathbb{G}_{\max }$ and $\mathbb{H}=\mathbb{H}_{\max }$. We will denote by a subscript $\mathbb{G}($ resp. $\mathbb{H})$ the object associated to $\mathbb{G}$ (resp. $\mathbb{H})$. Let $\pi$ be the surjective morphism from $C(\mathbb{G})$ to $C(\mathbb{H})$ which intertwines the comultiplications. Let $U$ be a unitary representation of $\widehat{\mathbb{H}}$ on a Hilbert space $K$ and suppose that $U$ has almost invariant vectors. Let $\rho$ be the unique morphism from $C(\mathbb{H})$ to $\mathcal{B}(K)$ such that $(\operatorname{id} \otimes \rho)\left(\mathcal{V}_{\mathbb{H}}\right)=U$. Consider the following unitary representation of $\widehat{\mathbb{G}}$ on $K: V=(\mathrm{id} \otimes(\rho \circ \pi))\left(\mathcal{V}_{\mathbb{G}}\right)$. We will show that $V$ has almost invariant vectors. Let $E \subset \operatorname{Irred}(\mathbb{G})$ be a finite subset and $\epsilon>0$. For $x \in \operatorname{Irred}(\mathbb{G})$ and $y \in$ $\operatorname{Irred}(\mathbb{H})$ denote by $u^{x} \in \mathcal{B}\left(H_{x}\right) \otimes C(\mathbb{G})$ and $v^{y} \in \mathcal{B}\left(H_{y}\right) \otimes C(\mathbb{H})$ a representative of $x$ and $y$ respectively. Note that $w^{x}=($ id $\otimes \pi)\left(u^{x}\right)$ is a finite dimensional unitary representation of $\mathbb{H}$, thus we can suppose that $w^{x}=\oplus n_{x, y} v^{y}$. Let $L=\left\{y \in \operatorname{Irred}(\mathbb{H}) \mid \exists x \in E, n_{x, y} \neq 0\right\}$. Because $U$ has almost invariant vectors, there exists a norm one vector $\xi \in K$ such that $\left\|U^{y} \eta \otimes \xi-\eta \otimes \xi\right\|<\epsilon\|\eta\|$ for all $y \in L$ and all $\eta \in H_{y}$. Using the isomorphism

$$
H_{x}=\bigoplus_{y \in \operatorname{Irred}(\mathbb{H I}), n_{x, y} \neq 0} \underbrace{\left(H_{y} \oplus \ldots \oplus H_{y}\right)}_{n_{x, y}},
$$

we can identify $V^{x}$ with $\oplus n_{x, y} U^{y}$ in $\bigoplus_{y} \mathcal{B}\left(H_{y}\right) \oplus \mathcal{B}\left(H_{y}\right) \oplus \ldots \oplus \mathcal{B}\left(H_{y}\right) \otimes \mathcal{B}(K)$. With this identification it is easy to see that, for all $x \in E$ and all $\eta$ in $H_{x}$, we have $\left\|V^{x} \eta \otimes \xi-\eta \otimes \xi\right\|<\epsilon\|\eta\|$. It follows that $V$ has almost invariant vectors and thus there is a non-zero $V$-invariant vector, say $l$, in $K$. To show that $l$ is also $U$-invariant it is sufficient to show that for every $y \in \operatorname{Irred}(\mathbb{H})$ there exists $x \in \operatorname{Irred}(\mathbb{G})$ such that $n_{x, y} \neq 0$. This follows from Lemma 1 .

Corollary 1. The discrete quantum groups $\widehat{A_{o}(n)}, \widehat{A_{u}(n)}$ and $\widehat{A_{s}(n)}$ do not have property $T$ for $n \geq 2$.

Proof. It follows directly from the preceding proposition and the following surjective morphisms:

$$
A_{o}(n) \rightarrow C^{*}\left(\star_{i=1}^{n} \mathbb{Z}_{2}\right), A_{u}(n) \rightarrow C^{*}\left(\mathbb{F}_{n}\right), A_{s}(n) \rightarrow C^{*}\left(\star_{i=1}^{n} \mathbb{Z}_{n_{i}}\right),
$$

where $\sum n_{i}=n$.

In the next Proposition we show that discrete quantum groups with property $T$ are unimodular.

Proposition 7. Let $\widehat{\mathbb{G}}$ be a discrete quantum group. If $\widehat{\mathbb{G}}$ has property $T$ then it is a Kac algebra, i.e. the Haar state $\varphi$ on $\mathbb{G}$ is a trace. 
Proof. Suppose $\widehat{\mathbb{G}}$ has property $T$ and let $\Gamma$ be the discrete group introduced in Proposition 2, Because $\operatorname{Sp}(\widehat{\delta})=\Gamma \cup\{0\}$ and $\hat{\Delta} \widehat{\delta}=\widehat{\delta} \otimes \widehat{\delta}$, we have an injective *-homomorphism

$$
\alpha: c_{0}(\Gamma) \rightarrow c_{0}(\widehat{\mathbb{G}}), \quad \alpha(f)=f(\widehat{\delta})
$$

satisfying $\Delta \circ \alpha=(\alpha \otimes \alpha) \circ \Delta_{\Gamma}$. By Proposition 6 , $\Gamma$ has property $T$. It follows that $\Gamma=\{1\}$ and $\widehat{\delta}=1$. Thus $Q_{x}=1$ for all $x \in \operatorname{Irred}(\mathbb{G})$. This means that $\varphi$ is a trace.

Proposition 8. Let $\widehat{\mathbb{G}}$ be a discrete quantum group. If $\widehat{\mathbb{G}}$ has property $T$ then it is finitely generated.

Proof. Let $\operatorname{Irred}(\mathbb{G})=\left\{x_{n} \mid n \in \mathbb{N}\right\}$ and $\mathcal{C}$ be the category of finite dimensional unitary representations of $\mathbb{G}$. For $i \in \mathbb{N}$ let $\mathcal{D}_{i}$ be the full subcategory of $\mathcal{C}$ generated by $\left(x_{0}, \ldots, x_{i}\right)$. This means that the irreducibles of $\mathcal{D}_{i}$ are the irreducible representations $u$ of $\mathbb{G}$ such that $u$ is equivalent to a sub-representation of $x_{k_{1}}^{\epsilon_{1}} \otimes \ldots \otimes x_{k_{l}}^{\epsilon_{l}}$ for $l \geq 1,0 \leq k_{j} \leq n$, and $\epsilon_{j}$ is nothing or the contragredient. The Hilbert spaces and the morphisms are the same in $\mathcal{D}_{i}$ or in $\mathcal{D}$. Thus we have $1_{\mathcal{C}} \in \mathcal{D}_{i}, \mathcal{D}_{i} \otimes \mathcal{D}_{i} \subset \mathcal{D}_{i}$ and $\overline{\mathcal{D}_{i}}=\mathcal{D}_{i}$. Let $\mathbb{H}_{i}$ be the compact quantum group such that $\mathcal{D}_{i}$ is the category of representation of $\mathbb{H}_{i}$. Let $U_{i} \in l^{\infty}\left(\widehat{\mathbb{G}} / \widehat{\mathbb{H}}_{i}\right) \otimes \mathcal{B}\left(l^{2}\left(\widehat{\mathbb{G}}^{\prime} \widehat{\mathbb{H}}_{i}\right)\right)$ be the quasi-regular representation of $\widehat{\mathbb{G}}$ modulo $\widehat{\mathbb{H}}_{i}$. Let $U$ be the direct sum of the $U_{i}$; this a unitary representation on $K=\bigoplus l^{2}\left(\widehat{\mathbb{G}}_{\mathbb{H}}\right)$. Let us show that $U$ has almost invariant vectors. Let $E \subset \operatorname{Irred}(\mathbb{G})$ be a finite subset. There exists $i_{0}$ such that $E \subset \operatorname{Irred}\left(\mathbb{H}_{i}\right)$ for all $i \geq i_{0}$. By Lemma 2 we have a unit vector $\xi$ in $l^{2}\left(\widehat{\mathbb{G}} / \widehat{\mathbb{H}}_{i}\right)$ such that $U_{i_{0}}^{x} \eta \otimes \xi=\eta \otimes \xi$ for all $x \in E$ and all $\eta \in H_{x}$. Let $\tilde{\xi}=\left(\xi_{i}\right) \in K$ where $\xi_{i}=0$ if $i \neq i_{0}$ and $\xi_{i_{0}}=\xi$. Then $\tilde{\xi}$ is a unit vector in $K$ such that $U^{x} \eta \otimes \tilde{\xi}=\eta \otimes \tilde{\xi}$ for all $x \in E$. It follows that $U$ has an almost invariant vector. By property $T$ there exists a non-zero invariant vector $l=\left(l_{i}\right) \in K$. There exists $m$ such that $l_{m} \neq 0$. Then $l_{m}$ is an invariant vector for $U_{m}$. By Lemma $3 \operatorname{Irred}(\mathbb{G}) / \operatorname{Irred}\left(\mathbb{H}_{m}\right)$ is a finite set. Let $y_{1}, \ldots, y_{l}$ be a complete set of representatives of $\operatorname{Irred}(\mathbb{G}) / \operatorname{Irred}\left(\mathbb{H}_{m}\right)$. Then $\mathcal{C}$ is generated by $\left\{y_{1}, \ldots, y_{l}, x_{0}, \ldots, x_{m}, \bar{x}_{0}, \ldots, \bar{x}_{m}\right\}$.

As in the classical case, we can show that property $T$ is equivalent to the existence of a Kazhdan pair.

Proposition 9. Let $\widehat{\mathbb{G}}$ be a finitely generated discrete quantum group. Let $E \subset \operatorname{Irred}(\mathbb{G})$ be a finite subset with $1 \in E$ such that $\mathcal{R}(\mathbb{G})$ is generated by $E$. The following assertions are equivalent:

1. $\widehat{\mathbb{G}}$ has property $T$.

2. There exists $\epsilon>0$ such that every unitary representation of $\widehat{\mathbb{G}}$ having an $(E, \epsilon)$-invariant vector has a non-zero invariant vector.

Proof. It is sufficient to show that 1 implies 2. Let $n \in \mathbb{N}^{*}$ and $E_{n}=\{y \in$ $\left.\operatorname{Irred}(\mathbb{G}) \mid y \subset x_{1} \ldots x_{n}, x_{i} \in E\right\}$. Because $1 \in E$, the sequence $\left(E_{n}\right)_{n \in \mathbb{N}^{*}}$ is increasing. Let us show that $\operatorname{Irred}(\mathbb{G})=\bigcup E_{n}$. Let $r \in \operatorname{Irred}(\mathbb{G})$. Because $\mathcal{R}(\mathbb{G})$ 
is generated by $E$, there exists a finite family of morphisms $b_{k} \in \operatorname{Mor}\left(r_{k}, r\right)$, where $r_{k}$ is a product of elements of $E$ and $\sum_{k} b_{k} b_{k}^{*}=I_{r}$. Let $L$ be the maximum of the length of the elements $r_{k}$. Because $1 \in E$, we can suppose that all the $r_{k}$ are of the form $x_{1} \ldots x_{L}$ with $x_{i} \in E$. Put $t_{k}=b_{k}^{*}$. Note that $t_{k}^{*} t_{k} \in \operatorname{Mor}(r, r)$. Because $r$ is irreducible and $\sum_{k} t_{k}^{*} t_{k}=I_{r}$, there exists a unique $k$ such that $t_{k}^{*} t_{k}=I_{r}$ and $t_{l}^{*} t_{l}=0$ if $l \neq k$. Thus $t_{k} \in \operatorname{Mor}\left(r, r_{k}\right)$ is an isometry. This means that $r \subset r_{k}=x_{1} \ldots x_{L}$, i.e. $r \in E_{L}$.

Suppose that $\widehat{\mathbb{G}}$ has property $T$ and 2 is false. Let $N=\operatorname{Max}\left\{n_{x} \mid x \in E\right\}$ and $\epsilon_{n}=\frac{1}{n^{2} \sqrt{N^{n}}}$. For all $n \in \mathbb{N}^{*}$ there exists a unitary representation $U_{n}$ of $\widehat{\mathbb{G}}$ on a Hilbert space $K_{n}$ with an $\left(E, \epsilon_{n}\right)$-invariant vector but without a non-zero invariant vector. Let $\xi_{n}$ be a unit vector in $K_{n}$ which is $\left(E, \epsilon_{n}\right)$-invariant. Write $U_{n}=\sum_{y \in \operatorname{Irred}(\mathbb{G})} U^{n, y}$ where $U^{n, y}$ is a unitary element in $\mathcal{B}\left(H_{y}\right) \otimes \mathcal{B}\left(K_{n}\right)$. Let us show the following:

$$
\left\|U^{n, y} \eta \otimes \xi_{n}-\eta \otimes \xi_{n}\right\|_{H_{y} \otimes K_{n}}<\frac{1}{n}\|\eta\|_{H_{y}}, \forall n \in \mathbb{N}^{*}, \forall y \in E_{n}, \forall \eta \in H_{y} .
$$

Let $y \in E_{n}$ and $t_{y} \in \operatorname{Mor}\left(y, x_{1} \ldots x_{n}\right)$ such that $t_{y}^{*} t_{y}=I_{y}$. Note that, by the definition of a representation and using the description of the coproduct on $\widehat{\mathbb{G}}$, we have $\left(t_{y} \otimes 1\right) U^{n, y}=U_{1, n+1}^{n, x_{1}} U_{2, n+1}^{n, x_{2}} \ldots U_{n, n+1}^{n, x_{n}}\left(t_{y} \otimes 1\right)$ where the subscripts are used for the leg numbering notation. It follows that, for all $\eta \in H_{y}$, we have:

$$
\begin{aligned}
\left\|U^{n, y} \eta \otimes \xi_{n}-\eta \otimes \xi_{n}\right\| & =\left\|\left(t_{y} \otimes 1\right) U^{n, y} \eta \otimes \xi_{n}-\left(t_{y} \otimes 1\right) \eta \otimes \xi_{n}\right\| \\
& =\left\|U_{1, n+1}^{n, x_{1}} U_{2, n+1}^{n, x_{2}} \ldots U_{n, n+1}^{n, x_{n}} t_{y} \eta \otimes \xi_{n}-t_{y} \eta \otimes \xi_{n}\right\| \\
& \leq \sum_{k=1}^{n}\left\|U_{k, n+1}^{n, x_{k}} t_{y} \eta \otimes \xi_{n}-t_{y} \eta \otimes \xi_{n}\right\| .
\end{aligned}
$$

Let $\left(e_{j}^{x_{i}}\right)_{1 \leq j \leq n_{x_{i}}}$ be an orthonormal basis of $H_{x_{i}}$ and put

$$
t_{y} \eta=\sum \lambda_{i_{1} \ldots i_{n}} e_{i_{1}}^{x_{1}} \otimes \ldots \otimes e_{i_{n}}^{x_{n}} .
$$

Then we have, for all $y \in E_{n}$ and $\eta \in H_{y}$,

$$
\begin{aligned}
\left\|U^{n, y} \eta \otimes \xi_{n}-\eta \otimes \xi_{n}\right\| & \leq \sum_{k}\left\|\sum_{i_{1} \ldots i_{n}} \lambda_{i_{1} \ldots i_{n}}\left(U_{k, n+1}^{n, x_{k}} e_{i_{1}}^{x_{1}} \otimes \ldots \otimes e_{i_{n}}^{x_{n}} \otimes \xi_{n}-e_{i_{1}}^{x_{1}} \otimes \ldots \otimes e_{i_{n}}^{x_{n}} \otimes \xi_{n}\right)\right\| \\
& \leq \sum_{k} \sum_{i_{1} \ldots i_{n}}\left|\lambda_{i_{1} \ldots i_{n}}\right|\left\|U^{n, x_{k}} e_{i_{k}}^{x_{k}} \otimes \xi_{n}-e_{i_{k}}^{x_{k}} \otimes \xi_{n}\right\| \\
& \leq n \epsilon_{n}\left\|t_{y} \eta\right\|_{1},
\end{aligned}
$$

where $\left\|t_{y} \eta\right\|_{1}=\sum\left|\lambda_{i_{1} \ldots i_{n}}\right|$. Note that $\left\|t_{y} \eta\right\|_{1} \leq \sqrt{N^{n}}|| \eta \|$, thus we have

$$
\begin{aligned}
\left\|U^{n, y} \eta \otimes \xi_{n}-\eta \otimes \xi_{n}\right\| & \leq n \epsilon_{n} \sqrt{N^{n}}\|\eta\| \\
& \leq \frac{1}{n}\|\eta\| .
\end{aligned}
$$


This proves Eq. (3). It is now easy to finish the proof. Let $U$ be the direct sum of the $U_{n}$. It is a unitary representation of $\widehat{\mathbb{G}}$ on $K=\bigoplus K_{n}$. Let $\delta>0$ and $L \subset \operatorname{Irred}(\mathbb{G})$ a finite subset. Because $\operatorname{Irred}(\mathbb{G})=\bigcup^{\uparrow} E_{n}$ there exists $n_{1}$ such that $L \subset E_{n}$ for all $n \geq n_{1}$. Choose $n \geq n_{1}$ such that $\frac{1}{n}<\delta$. Put $\xi=\left(0, \ldots, 0, \xi_{n}, 0, \ldots\right)$ where $\xi_{n}$ appears in the n-th place. Let $x \in L$ and $\eta \in H_{x}$. We have:

$$
\begin{aligned}
\left\|U^{x} \eta \otimes \xi-\eta \otimes \xi\right\| & =\left\|U^{n, x} \eta \otimes \xi_{n}-\eta \otimes \xi_{n}\right\| \\
& \leq \frac{1}{n}\|\eta\|<\delta\|\eta\| .
\end{aligned}
$$

Thus $U$ has almost invariant vectors. It follows from property $T$ that $U$ has a non-zero invariant vector, say $l=\left(l_{n}\right)$. There is a $n$ such that $l_{n} \neq 0$ and from the $\mathrm{U}$-invariance of $l$ we conclude that $l_{n}$ is $U_{n}$-invariant. This is a contradiction.

Such a pair $(E, \epsilon)$ as defined Proposition 9 is called a Kazhdan pair for $\widehat{\mathbb{G}}$. Let us give an obvious example of a Kazhdan pair.

Proposition 10. Let $\widehat{\mathbb{G}}$ be a finite-dimensional discrete quantum group. Then $\operatorname{Irred}(\mathbb{G}), \sqrt{2})$ is a Kazhdan pair for $\widehat{\mathbb{G}}$.

Proof. If $\widehat{\mathbb{G}}$ is finite-dimensional then it is compact, $\varphi$ is a trace and $\hat{\varphi}$ is a normal functional. For $x \in \operatorname{Irred}(\mathbb{G})$ let $\left(e_{i}^{x}\right)$ be an orthonormal basis of $H_{x}$ and $e_{i j}^{x}$ the associated matrix units. As $Q_{x}=1$, we have $\hat{\varphi}\left(e_{i j}^{x}\right)=\frac{\operatorname{dim}_{q}(x)^{2}}{n_{x}} \delta_{i j}$. Let $U \in l^{\infty}(\widehat{\mathbb{G}}) \otimes \mathcal{B}(K)$ be a unitary representation of $\widehat{\mathbb{G}}$ with a unit vector $\xi \in K$ such that:

$$
\operatorname{Sup}_{x \in \operatorname{Irred}(\mathbb{G}), 1 \leq j \leq n_{x}}\left\|U^{x} e_{j}^{x} \otimes \xi-e_{j}^{x} \otimes \xi\right\|<\sqrt{2} .
$$

Because $\hat{\varphi}(1)^{-1}(\hat{\varphi} \otimes \mathrm{id})(U)$ is the projection on the $U$-invariant vectors, $\tilde{\xi}=$ $(\hat{\varphi} \otimes \mathrm{id})(U) \xi \in K$ is invariant. Let us show that $\tilde{\xi}$ is non-zero. Writing $U^{x}=$ $\sum e_{i j}^{x} \otimes U_{i j}^{x}$ with $U_{i j}^{x} \in \mathcal{B}(K)$, we have:

$$
\left\|U^{x} e_{j}^{x} \otimes \xi-e_{j}^{x} \otimes \xi\right\|^{2}=2-2 \operatorname{Re}\left\langle U_{j j}^{x} \xi, \xi\right\rangle, \quad \text { for all } x \in \operatorname{Irred}(\mathbb{G}), 1 \leq j \leq n_{x} .
$$

It follows that $\operatorname{Re}\left\langle U_{j j}^{x} \xi, \xi\right\rangle>0$ for all $x \in \operatorname{Irred}(\mathbb{G})$ and all $1 \leq j \leq n_{x}$. Thus,

$$
\operatorname{Re}\langle\tilde{\xi}, \xi\rangle=\sum_{x, i, j} \operatorname{Re}\left(\hat{\varphi}\left(e_{i j}^{x}\right)\left\langle U_{i j}^{x} \xi, \xi\right\rangle\right)=\sum_{x, i} \frac{\operatorname{dim}_{q}(x)^{2}}{n_{x}} \operatorname{Re}\left(\left\langle U_{i i}^{x} \xi, \xi\right\rangle\right)>0 .
$$

Remark 4. It is easy to see that a discrete quantum group is amenable and has property $T$ if and only if it is finite-dimensional. Indeed, the existence of almost invariant vectors for the regular representation is equivalent with amenability and it is well known that a discrete quantum group is finite dimensional if and only if the regular representation has a non-zero invariant vector. Moreover the previous proposition implies that all finite-dimensional discrete quantum groups have property $T$. 
The main result of this paper is the following.

Theorem 3. Let $\widehat{\mathbb{G}}$ be discrete quantum group such that $L^{\infty}(\mathbb{G})$ is an infinite dimensional factor. The following assertions are equivalent:

1. $\widehat{\mathbb{G}}$ has property $T$.

2. $L^{\infty}(\mathbb{G})$ is a $\mathrm{II}_{1}$ factor with property $T$.

Proof. We can suppose that $\mathbb{G}$ is reduced, $C(\mathbb{G}) \subset \mathcal{B}\left(L^{2}(\mathbb{G})\right)$ and $\mathbb{V} \in l^{\infty}(\widehat{\mathbb{G}}) \otimes$ $\mathrm{E}^{\infty}(\mathbb{G})$. We denote by $M$ the von Neumann algebra $L^{\infty}(\mathbb{G})$. For each $x \in$ $\operatorname{Irred}(\mathbb{G})$ we choose an orthonormal basis $\left(e_{i}^{x}\right)_{1 \leq i \leq n_{x}}$ of $H_{x}$. When $\varphi$ is a trace we take $e_{i}^{\bar{x}}=J_{x}\left(e_{i}^{x}\right)$. We put $u_{i j}^{x}=\left(\omega_{e_{j}^{x}}, e_{i}^{x} \otimes \mathrm{id}\right)\left(u^{x}\right)$.

$1 \Rightarrow 2$ : Suppose that $\widehat{\mathbb{G}}$ has property $T$. By Proposition 7 , M is finite factor. Thus, it is a $\mathrm{II}_{1}$ factor. Let $(E, \epsilon)$ be a Kazhdan pair for $\widehat{\mathbb{G}}$. Let $K \in \mathcal{C}(M)$ with morphisms $\pi_{l}: M \rightarrow \mathcal{B}(K)$ and $\pi_{r}: M^{o p} \rightarrow \mathcal{B}(K)$. Let $\delta=\frac{\epsilon}{\operatorname{Max}\left\{n_{x} \sqrt{n_{x}}, x \in E\right\}}$. Suppose that there exists a unit vector $\xi^{\prime} \in K$ such that:

$$
\left\|u_{i j}^{x} \xi^{\prime}-\xi^{\prime} u_{i j}^{x}\right\|<\delta, \quad \forall x \in E, \forall 1 \leq i, j \leq n_{x} .
$$

Define $U=\left(\right.$ id $\left.\otimes \pi_{r}\right)\left(\mathbb{V}^{*}\right)\left(\right.$ id $\left.\otimes \pi_{l}\right)(\mathbb{V})$. Because $\mathbb{V}$ is a unitary representation of $\widehat{\mathbb{G}}$ and $\pi_{r}$ is an anti-homomorphism, it is easy to check that $U$ is a unitary representation of $\widehat{\mathbb{G}}$ on $K$. Moreover, for all $x \in E$, we have:

$$
\begin{aligned}
\left\|U^{x} e_{i}^{x} \otimes \xi^{\prime}-e_{i}^{x} \otimes \xi^{\prime}\right\| & =\left\|\left(\mathrm{id} \otimes \pi_{l}\right)\left(u^{x}\right) e_{i}^{x} \otimes \xi^{\prime}-\left(\mathrm{id} \otimes \pi_{r}\right)\left(u^{x}\right) e_{i}^{x} \otimes \xi^{\prime}\right\| \\
& =\left\|\sum_{k=1}^{n_{x}} e_{k}^{x} \otimes\left(u_{k i}^{x} \xi^{\prime}-\xi^{\prime} u_{k i}^{x}\right)\right\| \\
& \leq \sum_{k=1}^{n_{x}}\left\|e_{k}^{x} \otimes\left(u_{k i}^{x} \xi^{\prime}-\xi^{\prime} u_{k i}^{x}\right)\right\| \\
& <n_{x} \delta \leq \frac{\epsilon}{\sqrt{n_{x}}} .
\end{aligned}
$$

It follows easily that for all $x \in E$ and all $\eta \in H_{x}$ we have $\left\|U^{x} \eta \otimes \xi^{\prime}-\eta \otimes \xi^{\prime}\right\|<$ $\epsilon\|\eta\|$. Thus there exists a non-zero $U$-invariant vector $\xi \in K$. It is easy to check that $\xi$ is a central vector.

$2 \Rightarrow 1$ : Suppose that $M$ is a $\mathrm{II}_{1}$ factor with property $T$ and let $\epsilon>0$ and $b_{1}, \ldots, b_{n} \in M$ be as in Proposition 5 , Let $\varphi$ be the Haar state on $\mathbb{G}$. By [7, Theorem $8, \varphi$ is the unique tracial state on $M$. We can suppose that $\left\|b_{i}\right\|_{2}=1$. Using the classical G.N.S. construction $\left(L^{2}(\mathbb{G}), \Omega\right)$ for $\varphi$ we have, for all $a \in M$,

$$
a \Omega=\sum_{x, k, l} n_{x} \varphi\left(\left(u_{k l}^{x}\right)^{*} a\right) u_{k l}^{x} \Omega .
$$

In particular, $\left\|b_{i}\right\|_{2}^{2}=\sum n_{x}\left|\varphi\left(\left(u_{k l}^{x}\right)^{*} b_{i}\right)\right|^{2}=1$. Fix $\delta>0$ then there exists a finite subset $E \subset \operatorname{Irred}(\mathbb{G})$ such that, for all $1 \leq i \leq n$,

$$
\sum_{x \notin E, k, l} n_{x}\left|\varphi\left(\left(u_{k l}^{x}\right)^{*} b_{i}\right)\right|^{2}<\delta^{2} .
$$


Let $U$ be a unitary representation of $\widehat{\mathbb{G}}$ on $K$ having almost invariant vectors and $\xi \in K$ an $(E, \delta)$-invariant unit vector. Turn $L^{2}(\mathbb{G}) \otimes K$ into a correspondence from $M$ to $M$ using the morphisms $\pi_{l}: M \rightarrow \mathcal{B}\left(L^{2}(\mathbb{G}) \otimes K\right), \pi_{l}(a)=U(a \otimes 1) U^{*}$ and $\pi_{r}: M^{o p} \rightarrow \mathcal{B}\left(L^{2}(\mathbb{G}) \otimes K\right), \pi_{r}(a)=J a^{*} J \otimes 1$, where $J$ is the modular conjugation of $\varphi$. Let $\widehat{\xi}=\Omega \otimes \xi$. It is easy to see that $\pi_{l}\left(u_{k l}^{x}\right)=\sum_{s} u_{k s}^{x} \otimes U_{s l}^{x}$ and, for all $a \in M$,

$$
a \widehat{\xi}=\sum n_{x} \varphi\left(\left(u_{k l}^{x}\right)^{*} a\right) u_{k s}^{x} \Omega \otimes U_{s l}^{x} \xi
$$

Note that, because $\varphi$ is a trace, $\Omega$ is a central vector in $L^{2}(\mathbb{G})$ and we have, for all $a \in M, \widehat{\xi} a=a \Omega \otimes \xi$. It follows that, for all $1 \leq i \leq n$, we have

$$
\begin{aligned}
\left\|b_{i} \widehat{\xi}-\widehat{\xi} b_{i}\right\|^{2} & =\left\|\sum_{x, k, l, s} n_{x} \varphi\left(\left(u_{k l}^{x}\right)^{*} b_{i}\right) u_{k s}^{x} \Omega \otimes U_{s l}^{x} \xi-\sum_{x, k, l} n_{x} \varphi\left(\left(u_{k l}^{x}\right)^{*} b_{i}\right) u_{k l}^{x} \Omega \otimes \xi\right\|^{2} \\
& =\left\|\sum_{x, k, l} n_{x} \varphi\left(\left(u_{k l}^{x}\right)^{*} b_{i}\right)\left(\sum_{s} u_{k s}^{x} \Omega \otimes U_{s l}^{x} \xi-u_{k l}^{x} \Omega \otimes \xi\right)\right\|^{2} \\
& =\left\|\sum_{x, k, l} \sqrt{n_{x}} \varphi\left(\left(u_{k l}^{x}\right)^{*} b_{i}\right)\left(\sum_{s} e_{s}^{x} \otimes J_{x}\left(e_{k}^{x}\right) \otimes U_{s l}^{x} \xi-e_{l}^{x} \otimes J_{x}\left(e_{k}^{x}\right) \otimes \xi\right)\right\|^{2} \\
& =\left\|\sum_{x, k, l} \sqrt{n_{x}} \varphi\left(\left(u_{k l}^{x}\right)^{*} b_{i}\right) J_{x}\left(e_{k}^{x}\right) \otimes\left(\sum_{s} e_{s}^{x} \otimes U_{s l}^{x} \xi-e_{l}^{x} \otimes \xi\right)\right\|^{2} \\
& =\left\|\sum_{x, k, l} \sqrt{n_{x}} \varphi\left(\left(u_{k l}^{x}\right)^{*} b_{i}\right) J_{x}\left(e_{k}^{x}\right) \otimes\left(U^{x} e_{l}^{x} \otimes \xi-e_{l}^{x} \otimes \xi\right)\right\|^{2} \\
& =\sum_{x, k} n_{x}\left\|\sum_{l} \varphi\left(\left(u_{k l}^{x}\right)^{*} b_{i}\right)\left(U^{x} e_{l}^{x} \otimes \xi-e_{l}^{x} \otimes \xi\right)\right\|^{2} \\
& \left.=\sum_{x, k} n_{x} \| U^{x} \eta_{k}^{x} \otimes \xi-\eta_{k}^{x} \otimes \xi\right) \|^{2}, \quad \text { where } \eta_{k}^{x}=\sum_{l} \varphi\left(\left(u_{k l}^{x}\right)^{*} b_{i}\right) e_{l}^{x} \\
& \left.\left.=\sum_{x \in E, k} n_{x} \| U^{x} \eta_{k}^{x} \otimes \xi-\eta_{k}^{x} \otimes \xi\right)\left\|^{2}+\sum_{x \notin E, k} n_{x}\right\| U^{x} \eta_{k}^{x} \otimes \xi-\eta_{k}^{x} \otimes \xi\right) \|^{2} \\
& <\delta^{2} \sum_{x \in E, k} n_{x}\left\|\eta_{k}^{x}\right\|^{2}+4 \sum_{x \notin E, k} n_{x}\left\|\eta_{k}^{x}\right\|^{2} \\
& <\delta^{2} \sum_{x \in E, k, l} n_{x}\left|\varphi\left(\left(u_{k l}^{x}\right)^{*} b_{i}\right)\right|^{2}+4 \sum_{x \notin E, k, l} n_{x}\left|\varphi\left(\left(u_{k l}^{x}\right)^{*} b_{i}\right)\right|^{2} \\
& <\delta^{2}+4 \delta^{2}=5 \delta^{2} .
\end{aligned}
$$

By Proposition 5, for $\delta$ small enough, there exists a central unit vector $\widehat{\eta} \in$ $L^{2}(\mathbb{G}) \otimes K$ with $\|\widehat{\eta}-\widehat{\xi}\|<\sqrt{5} C \delta$. Let $P$ be the orthogonal projection on $\mathbb{C} \Omega$. If $\delta$ is small enough then there is a non-zero $\eta \in K$ such that $(P \otimes 1) \widehat{\eta}=\Omega \otimes \eta$. Write $\widehat{\eta}=\sum_{y, s, t} e_{t}^{y} \otimes e_{s}^{\bar{y}} \otimes \eta_{s, t}^{y}$ where $\eta_{s, t}^{y} \in K$ and $\eta^{1}=\eta$. We have, for all 
$x \in \operatorname{Irred}(\mathbb{G})$ and all $1 \leq i, j \leq n_{x}, \pi_{l}\left(u_{i j}^{x}\right) \widehat{\eta}=\pi_{r}\left(u_{i j}^{x}\right) \widehat{\eta}$. This means:

$$
\sum_{k, y, t, s} u_{i k}^{x}\left(e_{t}^{y} \otimes e_{s}^{\bar{y}}\right) \otimes U_{k j}^{x} \eta_{s, t}^{y}=\sum_{y, t, s} J\left(u_{i j}^{x}\right)^{*} J\left(e_{t}^{y} \otimes e_{s}^{\bar{y}}\right) \otimes \eta_{s, t}^{y} .
$$

Let $Q$ be the orthogonal projection on $H_{x} \otimes H_{\bar{x}}$. Using

$$
u_{i k}^{x}\left(e_{t}^{y} \otimes e_{s}^{\bar{y}}\right) \subset \bigoplus_{z \subset x \otimes y} H_{z} \otimes H_{\bar{z}},
$$

and $x \subset x \otimes y$ if and only if $y=1$, we find:

$$
Q u_{i k}^{x}\left(e_{t}^{y} \otimes e_{s}^{\bar{y}}\right)=\delta_{y, 1} \frac{1}{\sqrt{n_{x}}} e_{k}^{x} \otimes e_{i}^{\bar{x}}
$$

Using the same arguments and the fact that $J=\bigoplus\left(J_{x} \otimes J_{\bar{x}}\right)$ we find:

$$
Q J\left(u_{i j}^{x}\right)^{*} J\left(e_{t}^{y} \otimes e_{s}^{\bar{y}}\right)=\delta_{y, 1} \frac{1}{\sqrt{n_{x}}} e_{j}^{x} \otimes e_{i}^{\bar{x}} .
$$

Applying $Q \otimes 1$ to Eq. (4) we obtain:

$$
\sum_{k} e_{k}^{x} \otimes e_{i}^{\bar{x}} \otimes U_{k j}^{x} \eta=e_{j}^{x} \otimes e_{i}^{\bar{x}} \otimes \eta, \quad \text { for all } x \in \operatorname{Irred}(\mathbb{G}), 1 \leq i, j \leq n_{x} .
$$

Thus, for all $x \in \operatorname{Irred}(\mathbb{G})$ and all $1 \leq j \leq n_{x}$, we have:

$$
U^{x}\left(e_{j}^{x} \otimes \eta\right)=\sum_{k} e_{k}^{x} \otimes U_{k j}^{x} \eta=e_{j}^{x} \otimes \eta .
$$

Thus $\eta$ is a non-zero $U$-invariant vector.

The preceding theorem admits the following corollary about the persistance of property $T$ by twisting.

Corollary 2. Let $\mathbb{G}$ be a compact quantum group such that $L^{\infty}(\mathbb{G})$ is an infinite dimensional factor. Suppose that $K$ is an abelian co-subgroup of $\mathbb{G}$ (see [8]). Let $\sigma$ be a continuous bicharacter on $\widehat{K}$ and denote by $\mathbb{G}^{\sigma}$ the twisted quantum group. If $\widehat{\mathbb{G}}$ has property $T$ then $\widehat{\mathbb{G}^{\sigma}}$ is a discrete quantum group with property $T$.

Proof. If $\widehat{\mathbb{G}}$ has property $T$ then the Haar state $\varphi$ on $\mathbb{G}$ is a trace. Thus the co-subgroup $K$ is stable (in the sense of [8]) and the Haar state $\varphi_{\sigma}$ on $\mathbb{G}^{\sigma}$ is the same, i.e. $\varphi=\varphi_{\sigma}$. It follows that $\mathbb{G}^{\sigma}$ is a compact quantum group with $L^{\infty}\left(\mathbb{G}^{\sigma}\right)=L^{\infty}(\mathbb{G})$. Thus $L^{\infty}\left(\mathbb{G}^{\sigma}\right)$ is a $\mathrm{II}_{1}$ factor with property $T$ and $\widehat{\mathbb{G}_{\sigma}}$ has property $T$.

Example 1. The group $S L_{2 n+1}(\mathbb{Z})$ is I.C.C. and has property $T$ for all $n \geq 1$. Let $K_{n}$ be the subgroup of diagonal matrices in $S L_{2 n+1}(\mathbb{Z})$. We have $K_{n}=$ $\mathbb{Z}_{2}^{2 n}=\left\langle t_{1}, \ldots, t_{2 n} \mid t_{i}^{2}=1 \forall i, t_{i} t_{j}=t_{j} t_{i} \forall i, j\right\rangle$ and $K_{n}$ is an abelian co-subgroup 
of $\mathbb{G}_{2 n+1}=\left(C^{*}\left(S L_{2 n+1}(\mathbb{Z})\right), \Delta\right)$. Consider the following bicharacter on $\widehat{K_{n}}=$ $K_{n}: \sigma$ is the unique bicharacter such that $\sigma\left(t_{i}, t_{j}\right)=-1$ if $i \leq j$ and $\sigma\left(t_{i}, t_{j}\right)=1$

if $i>j$. By the preceding Corollary, the twisted quantum group $\widetilde{\mathbb{G}_{2 n+1}^{\sigma}}$ has property $T$ for all $n \geq 1$. When $n$ is even, $S L_{n}(\mathbb{Z})$ is not I.C.C. and $I$ and $-I$ lie in the centre of $S L_{n}(\mathbb{Z})$. We consider the group $P S L_{n}(\mathbb{Z})=S L_{n}(\mathbb{Z}) /\{I,-I\}$ in place of $S L_{n}(\mathbb{Z})$ in the even case. It is well known that $P S L_{2 n}(\mathbb{Z})$ is I.C.C. and has property $T$ for $n \geq 2$. The group of diagonal matrices in $S L_{2 n}(\mathbb{Z})$ is $\mathbb{Z}_{2}^{2 n-1}$ which contains $\{I,-I\}$. We consider the following abelian subgroup of $P S L_{2 n}(\mathbb{Z}): L_{n}=\mathbb{Z}_{2}^{2 n-1} /\{I,-I\}=\mathbb{Z}_{2}^{2 n-2}=K_{n-1}$ and the same bicharacter $\sigma$ on $K_{n-1}$. Let $\mathbb{G}_{2 n}=\left(C^{*}\left(P S L_{2 n}(\mathbb{Z})\right), \Delta\right)$. By the preceding Corollary, the twisted quantum group $\widehat{\mathbb{G}_{2 n}^{\sigma}}$ has property $T$ for all $n \geq 2$.

\section{References}

[1] T. Banica, Théorie des représentations du groupe quantique compact libre O(n), C. R. Acad. Sci. Paris Sér. I Math. 322 no. 3 (1996), 241-244.

[2] T. Banica, Le groupe quantique compact libre U(n), Comm. Math. Phys. 190 no. 1 (1997), 143-172.

[3] T. Banica and R. Vergnioux, Growth estimates for discrete quantum groups, J. Noncommut. Geom., to appear.

[4] A. Connes and V. Jones, Property T for von Neumann algebras, Bull. London Math. Soc. 17 no. 1 (1985), 57-62.

[5] V.G. Drinfeld, Quantum groups, Proceedings of the International Congress of Mathematicians, 1, 2 Berkeley, Calif. (1986), pp. 798-820, Amer. Math. Soc., Providence, RI (1987).

[6] M. Enock, Sous-facteurs intermédiaires et groupes quantiques mesurés, $J$. Operator Theory 42 no. 2 (1999), 305-330.

[7] P. Fima, On locally compact quantum groups whose algebras are factors. $J$. Funct. Anal. 244 no. 1 (2007), 78-94.

[8] P. Fima and L. Vainerman, Twisting and Rieffel's deformation of locally compact quantum groups. Deformation of the Haar measure, Comm. Math. Phys., to appear.

[9] M. Jimbo, A q-difference analogue of $\mathrm{U}(\mathrm{g})$ and the Yang-Baxter equation, Lett. Math. Phys. 10 no. 1 (1985), 63-69.

[10] J. Kustermans, Induced corepresentations of locally compact quantum groups, J. Funct. Anal. 194 no. 2 (2002), 410-459.

[11] J. Kustermans and S. Vaes, Locally compact quantum groups, Ann. Sci. École Norm. Sup. (4) 33 no. 6 (2000), 837-934. 
[12] A. Maes and A. Van Daele, Notes on Compact Quantum Groups, Nieuw Arch. Wisk. (4) 16 no. 1-2 (1998), 73-112.

[13] M. Rosso, Algèbres enveloppantes quantifiées, groupes quantiques compacts de matrices et calcul différentiel non commutatif., Duke Math. J. 61 no. 1 (1990), 11-40.

[14] R.Tomatsu, Amenable discrete quantum groups, J. Math. Soc. Japan 58 no. 4 (2006), 949-964.

[15] S. Vaes and R. Vergnioux, The boundary of universal discrete quantum groups, exactness and factoriality, Duke Math. J. 140 no. 1 (2007), 35-84.

[16] S. Vaes, The unitary implementation of a locally compact quantum group action, J. Funct. Anal. 180 no. 2 (2001),426-480.

[17] R. Vergnioux, K-amenability for amalgamated free products of amenable discrete quantum groups, J. Funct. Anal. 212 no. 1 (2004), 206-221.

[18] A. Van Daele and S. Wang, Universal quantum groups, Internat. J. Math. 7 no. 2 (1996), 255-263.

[19] S.L. Woronowicz, Compact matrix pseudogroup, Comm. Math. Phys. 111 no. 4 (1987), 613-665.

[20] S.L. Woronowicz, Tannaka-Krein duality for compact matrix pseudogroups. Twisted SU(N) groups, Invent. Math. 93 no. 1 (1988), 35-76.

[21] S.L. Woronowicz, Compact quantum groups, Symétries quantiques, Les Houches (1995), pp. 845-884, North-Holland, Amsterdam (1998). 